\title{
Refuge
}

Canada's Journal on Refugees

Revue canadienne sur les réfugiés

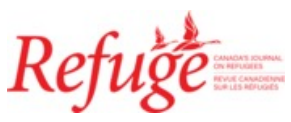

\section{Considérations en matière d'éthique de la recherche auprès de personnes en situation de migration forcée}

\section{Christina Clark-Kazak}

Volume 33, Number 2, 2017

URI: https://id.erudit.org/iderudit/1043058ar

DOI: https://doi.org/10.7202/1043058ar

See table of contents

Publisher(s)

Centre for Refugee Studies, York University

ISSN

0229-5113 (print)

1920-7336 (digital)

Explore this journal

Cite this document

Clark-Kazak, C. (2017). Considérations en matière d'éthique de la recherche auprès de personnes en situation de migration forcée. Refuge, 33(2), 3-10. https://doi.org/10.7202/1043058ar
Article abstract

La recherche peut contribuer à une meilleure compréhension de l'expérience des personnes en situation de migration forcée et appuyer ainsi l'élaboration de politiques et de programmes pertinents; toutefois cette recherche peut aussi contraindre et causer des préjudices pour les répondants[1] à la recherche. Dans les situations de migration forcée, les enjeux sont considérables en raison du statut juridique précaire des répondants, des relations de pouvoir inégal, de la vaste portée des lois antiterroristes et de la criminalisation de la migration. Face à cette situation, le Conseil canadien pour les réfugiés, le Centre pour les études sur les réfugiés (Centre for Refugee Studies) de l'Université York et l'Association canadienne d'études sur les réfugiés et la migration forcée (ACERMF) ont travaillé ensemble pour élaborer les considérations particulières en matière d'éthique de la recherche auprès de personnes en situation de migration forcée. Ces considérations complètent les principes éthiques existants. Elles présentent un intérêt pour toutes les personnes qui participent à la collecte d'informations - dans un cadre universitaire ou communautaire et pour les personnes qui prennent part à la recherche. Ce document présente nos principes directeurs ainsi que les concepts éthiques du consentement libre et éclairé, du respect de la vie privée et de l'analyse coûts-avantages.
Copyright (c) Refuge: Canada's Journal on Refugees, 2017

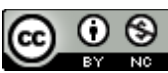

This document is protected by copyright law. Use of the services of Érudit (including reproduction) is subject to its terms and conditions, which can be viewed online.

https://apropos.erudit.org/en/users/policy-on-use/ 


\title{
Considérations en matière déthique de la recherche auprès de personnes en situation de migration forcée
}

\author{
CHRISTINA CLARK-KAZAK, AVEC LE CONSEIL CANADIEN POUR LES RÉFUGIÉS, \\ L'ASSOCIATION CANADIENNE D'ÉTUDES SUR LES RÉFUGIÉS ET LA MIGRATION FORCÉE, \\ ET LE CENTRE POUR LES ÉTUDES SUR LES RÉFUGIÉS DE L'UNIVERSITÉ YORK ${ }^{1}$
}

\section{Préambule}

La recherche auprès de personnes en situation de migration forcée nous permet de mieux comprendre leurs expériences et pourrait guider les prises de décision fondées sur des données probantes. Cette recherche crée aussi des opportunités et des défis particuliers en matière déthique. Le présent document vise à fournir aux chercheurs ${ }^{2}$, aux organismes communautaires et aux personnes en situation de migration forcée, des informations sur les particularités des contextes de la migration forcée. Ces informations compléteront les principes et les cadres existants en matière déthique de la recherche auprès de sujets humains en général. Elles s'appuient sur les bonnes pratiques recensées lors d'un examen des documents provenant de la société civile, des gouvernements et de la littérature savante (voir à l’annexe 3 la liste des ressources).

Les contextes de la recherche sur la migration forcée sont particuliers pour les raisons suivantes :

1. Les personnes qui fuient en traversant des frontières ont des droits juridiques et des opportunités qui diffèrent de ceux des personnes nées dans le pays d'accueil. Le droit de rester dans le pays d'accueil peut être révoqué ou menacé sur la base de données recueillies pendant la recherche. Étant donné ce statut juridique précaire, les chercheurs qui travaillent avec des personnes en situation de migration forcée doivent bien connaitre leurs obligations en matière d'éthique, pour minimiser les risques.
2. Les personnes déplacées sont souvent dans des situations où existent des relations de pouvoir inégal, sachant que leur survie ou leur statut juridique dépend de parrains, de fournisseurs de services ou du gouvernement. Cette situation extrême de dépendance peut remettre en question le caractère volontaire du consentement à participer à une recherche menée par ces organismes ou en partenariat avec ceux-ci.

3. Sachant que des groupes sont réputés mener des activités terroristes ${ }^{3}$ dans les pays dorigine ou d'asile, la recherche pourrait entrer en conflit avec des lois antiterroristes. Par exemple, les répondants à la recherche pourraient divulguer des liens avec des groupes considérés comme ayant des objectifs terroristes, ou exprimer leur sympathie à leur égard. Comme les chercheurs ne sont pas protégés par les mêmes privilèges juridiques que les médecins et les avocats, il pourrait y avoir des limites à la confidentialité des informations communiquées, ce qui pose des défis sur le plan éthique.

4. Pendant tout le processus de demande d'asile, les personnes déplacées sont amenées à raconter plusieurs fois leur histoire : à des représentants de l'immigration, à des membres des professions juridiques, à des fournisseurs de services et à des décideurs. Les questions des chercheurs pourraient accroittre la peine qu'ont les personnes déplacées à raconter des situations douloureuses de conflits, de violence, de violations et d'abus. 
5. Bien que la recherche universitaire puisse contribuer à améliorer les politiques et les programmes pour les personnes en situation de migration forcée, les résultats de la recherche ne sont pas toujours partagés, de façon accessible et en temps opportun, avec les personnes déplacées et avec les partenaires hors du milieu universitaire.

\section{Définitions}

Dans le cadre de ces directives, la recherche est définie comme toute activité comprenant la collecte de données et la création de savoir, en collaboration avec les personnes déplacées. Cette activité inclut, sans s'y limiter, les entrevues, les groupes de discussion, les sondages, les expériences, les observations, ainsi que l'accès à des dossiers, à des données administratives et à des auto-ethnographies comprenant la collecte de données par des tiers. Il convient de noter que toutes les activités de recherche ne doivent pas nécessairement être approuvées par un comité d'éthique de la recherche; cependant, ces directives établissent des principes de bonne pratique dont on devrait tenir compte lors des recherches dans des contextes de migration forcée.

«Les personnes en situation de migration forcée» comprennent un large éventail de personnes déplacées, dont des demandeurs du statut de réfugié, des détenteurs du statut de réfugié, des personnes dont les demandes de statut de réfugié ont été rejetées, des victimes de la traite des personnes et des personnes déplacées à l'intérieur de leur pays. Ces directives s'appliquent à tous les groupes qui ont été obligés de quitter leur maison, et pas seulement aux détenteurs du statut de réfugié.

\section{Principes directeurs}

Notre recherche sur les personnes en situation de migration forcée sera guidée par les principes fondamentaux suivants:

\section{Équité}

Nous nous efforcerons de rendre les relations, lors de la recherche, le plus équitables possible, en ayant conscience de la dynamique du pouvoir et en prévenant les risques d'abus de pouvoir.

- Nous choisirons les répondants et les équipes de recherche en fonction de principes équitables.

- Nous inclurons une diversité de perspectives et éviterons les biais basés sur le genre, l'orientation sexuelle, l'âge, la capacité, la religion, la culture, l'ethnicité ou la nationalité.

- Nous aurons conscience de notre position, de nos préjugés et de notre responsabilité dans le cadre du processus de recherche.
- Nous chercherons à établir des relations basées sur la confiance mutuelle.

\section{Droit à l'autodétermination}

Nous respecterons et appuierons le droit des personnes en situation de migration forcée à prendre leurs propres décisions en ce qui concerne leur vie et leur niveau de participation à la recherche.

- Nous privilégierons les droits et le bien-être des répondants par rapport aux objectifs de la recherche.

- Nous respecterons la dignité des répondants dans la manière dont nous les présenterons - individuellement et collectivement.

\section{Compétence}

Nous respecterons notre obligation d'agir avec compétence.

- Nous choisirons des méthodes de recherche appropriées.

- Nous acquerrons une compréhension appropriée de la culture et de la diversité.

- Nous sélectionnerons, formerons et superviserons les assistants de recherche et les interprètes.

- Nous fournirons aux répondants des informations exactes sur leurs droits.

- Nous reconnaîtrons nos limites et orienterons vers les personnes ou les services appropriés les répondants qui démontrent des besoins ou demandent de l'information.

- Nous accepterons notre obligation de diligence.

\section{Partenariat}

Nous inclurons les partenaires pertinents pour notre recherche dans tout le processus de recherche: depuis la conception de la recherche et la collecte de données jusquà l'analyse et la diffusion des résultats.

- Nous élaborerons des protocoles et des mécanismes appropriés pour permettre la pleine participation des partenaires pertinents.

- Nous déterminerons à l'avance des mécanismes de résolution de problèmes culturellement appropriés, qui serviront en cas de désaccords concernant les méthodes, la conception ou la diffusion de la recherche.

- Nous prônerons la copropriété de la recherche et reconnaitrons de manière respectueuse la contribution de chaque partenaire. S'il n'est pas possible de mentionner les coauteurs, par exemple lorsque les étudiants doivent écrire seuls des travaux ou des dissertations pour répondre aux exigences dobtention de leur diplôme, ceci sera clairement expliqué à tous les participants à la recherche, dès le début du processus de recherche. 


\section{Application des principes éthiques fondamentaux, à la recherche dans des contextes de migration}

\section{forcée}

\section{Consentement libre et éclairé}

Tous les répondants doivent consentir, de manière libre et éclairée, à participer à la recherche après avoir été informés des risques et des avantages potentiels liés à leur participation. Ils doivent pouvoir se retirer de la recherche en tout temps. Les éléments suivants doivent être pris en considération lorsquon cherche à obtenir le consentement libre et éclairé de personnes en situation de migration forcée:

- Les personnes déplacées qui ont eu des interactions négatives avec les autorités ou qui ont des traditions culturelles particulières pourraient se méfier des formulaires de consentement écrit. Dans ces situations, un consentement oral devrait aussi être possible et des procédures claires sur la façon d’obtenir et d'enregistrer ce consentement oral devraient être fournies.

- Les chercheurs comptent parfois sur les fournisseurs de services ou sur les organismes d'aide aux réfugiés pour avoir accès à des répondants potentiels. Cependant, seul le répondant peut consentir à participer. Lorsque l'accès a été fourni par un «gardien», il est important que les répondants potentiels comprennent qu'ils ont le droit de refuser de participer à la recherche à n'importe quelle étape du processus, et que ce refus n'aura aucune conséquence sur les services ou le niveau d'aide qu'ils reçoivent. Il est aussi important de réfléchir à la façon dont l'anonymat des participants sera garanti étant donné que leur nom a été fourni par un organisme ou un fournisseur de services. Cet aspect doit être clairement expliqué dans le protocole et dans le processus de consentement.

- Bien qu'une compensation financière puisse être offerte pour dédommager les répondants de leur temps, des frais de garde ou des coûts de transport - et éliminer ainsi les obstacles à leur participation - cette compensation devrait être raisonnable et proportionnelle aux dépenses. Les personnes déplacées ayant des besoins financiers ne devraient pas se sentir contraintes de participer à la recherche pour des raisons financières. La compensation ne doit pas être liée au fait de participer jusqu'au bout à la recherche.

- La recherche profite rarement directement aux répondants eux-mêmes. Ceci doit être clairement expliqué aux personnes en situation de migration forcée, afin quelles ne participent pas seulement dans l'espoir de recevoir directement des avantages matériels, juridiques ou autres.
- Les répondants doivent être informés des risques sur les plans financier, émotif, social/communautaire ou autre, associés à leur participation, et particulièrement des limites de la confidentialité en cas de divulgation de criminalité ou d’automutilation, comme précisé ci-après.

\section{Confidentialité et respect de la vie privée}

Les chercheurs ont le devoir de protéger les renseignements personnels des répondants et de ne communiquer aucune caractéristique d'identification qui compromettrait lanonymat, particulièrement si léchantillon est petit. Plus particulièrement, il faudrait tenir compte des considérations suivantes pour la recherche dans des contextes de migration forcée:

- La protection juridique des chercheurs est limitée quand des tiers utilisent des assignations à témoigner dans des procédures criminelles ou des poursuites civiles. Cette question est particulièrement importante étant donné la criminalisation de la migration et la vaste portée des lois antiterroristes. Les répondants devraient être informés de ces risques. Les chercheurs devraient éviter de recueillir des données potentiellement incriminantes et se limiter à des données directement pertinentes pour le sujet de la recherche. Ces données devraient être rendues totalement anonymes immédiatement après leur collecte afin qu'aucune information ne puisse être directement liée à une personne en particulier. Dans la mesure du possible, les chercheurs devraient éviter de recueillir des données contenant des identificateurs personnels.

- Les chercheurs et les répondants devraient aussi savoir que les outils de sondage électroniques, dont les serveurs sont hébergés à lextérieur du Canada, sont soumis aux lois des pays tiers, lesquelles peuvent inclure, dans certains cas, l'accès à toutes les données recueillies. $\mathrm{Si}$ c'est le cas, le formulaire de consentement devrait inclure des renseignements sur l'accès, le stockage et les limites potentielles de la confidentialité.

- Dans certains cas, les répondants révéleront spontanément des situations préjudiciables, comme des abus, de l'exploitation ou de l'automutilation. Le chercheur devra alors clairement expliquer les limites de la confidentialité, particulièrement s'il existe des normes disciplinaires prévoyant une obligation de signalement pour les chercheurs. Il faudra aussi avoir une stratégie pour orienter les répondants vers des ressources externes lorsque de telles situations sont révélées.

- Dans certains cas, l'identification des répondants à la recherche peut avoir de graves répercussions sur leur sécurité, leur bien-être, leur statut de migrant et 
leur admissibilité à des services. La confidentialité des informations est alors cruciale. Les chercheurs devraient s'assurer de crypter les données et de les conserver de manière sécurisée; ils devraient aussi retirer toute caractéristique qui pourrait identifier le répondant, notamment par association.

- Bien que les documents audiovisuels puissent être des données importantes, ces documents posent des difficultés particulières en ce qui concerne la confidentialité et l'anonymat. Ils devraient être utilisés avec précaution, et seulement avec la permission explicite de toutes les personnes apparaissant dans les documents. Les répondants à la recherche devraient pouvoir demander, à n'importe quel moment, la destruction des documents audiovisuels dans lesquels ils apparaissent.

- Les interprètes, les chercheurs et toutes les autres personnes qui participent au processus de recherche doivent signer une entente de confidentialité.

- Lorsque les répondants à la recherche souhaitent que leur nom apparaisse dans la recherche, les chercheurs doivent respecter ce désir d’autodétermination et trouver un moyen d'y répondre, sans compromettre l'anonymat des personnes qui ne veulent pas être identifiées.

\section{Minimiser les risques de préjudice et maximiser les} retombées de la recherche

- Les chercheurs devraient s'appuyer sur des recherches semblables et y collaborer, pour éviter des recherches excessives sur certaines populations.

- Les chercheurs devraient éviter les sujets délicats et qui peuvent conduire à un nouveau traumatisme - comme la violence sexuelle et la torture - sauf s'ils sont directement liés au sujet de la recherche. Dans ces cas, des ressources et des services externes doivent avoir été identifiés et être disponibles si un aiguillage est nécessaire.

- Il faut tout mettre en œuvre pour inclure une diversité de perspectives dans les études, et adopter une méthodologie et des stratégies de recrutement précises afin de tenir compte d'un éventail de perspectives et de besoins en matière de recherche, basés sur l'âge, le genre, la sexualité, la capacité, la classe, la race, léducation, l'alphabétisation et la langue.

- Les chercheurs devraient s'assurer de diffuser largement les résultats de leurs recherches dans les langues pertinentes, par le biais de divers médias (oral, écrit, visuel), et ils devraient indiquer clairement aux répondants où les résultats seront disponibles. 
Annexe 1: Liste de vérification pour les chercheurs

1. Ai-je besoin d'une approbation déontologique pour ce projet? Si oui, comment puis-je l'obtenir?

2. Le cas échéant, ai-je partagé mon protocole en matière d'éthique avec les partenaires concernés?

3. Qui profitera de cette recherche?

4. Qui d'autre fait de la recherche sur ce sujet et auprès de quelle population? Avons-nous coordonné nos activités pour éviter des recherches excessives?

5. Quelles sont les limites potentielles de la confidentialité? Quelles stratégies ai-je mises en place au cas où lon me révèlerait des situations de criminalité, d'exploitation ou d'automutilation?

6. Qui nest pas inclus dans ma proposition de recherche? Quelles stratégies puis-je utiliser pour faciliter la participation de ces personnes?

7. Comment inclurai-je les partenaires concernés dans toutes les phases de mon projet, depuis sa conception jusquà la diffusion des résultats? Quels mécanismes et quels protocoles sont en place pour assurer leur pleine participation?

8. Ai-je prévu dans le budget de mon projet une compensation pour le temps et les autres ressources que les partenaires non universitaires investiront dans la recherche, notamment les répondants, les membres des comités consultatifs, les recruteurs de répondants et les personnes qui facilitent la participation d'autres répondants?
Annexe 2: Liste de vérification pour les organisations et les personnes qui travaillent dans des contextes de migration forcée et qui sont contactées pour participer à une recherche

1. S'il y a lieu, le chercheur a-t-il obtenu l'approbation déontologique de son établissement d'attache? S’il n'existe pas de processus d'approbation déontologique, comment les principes du consentement, de la confidentialité et de la réduction des préjudices seront-ils évalués et appliqués?

2. Le chercheur a-t-il adopté un processus approprié de consentement libre et éclairé?

3. Comment communiquerons-nous avec nos clients et nos collègues pour qu'ils comprennent qu'ils ne sont pas obligés de participer à la recherche pour continuer à recevoir nos services?

4. Comment le respect de la vie privée et la confidentialité des données seront-ils assurés?

5. Quels sont les processus en place si la recherche révèle des situations de criminalité, d'exploitation ou d'automutilation?

6. Qui nest pas inclus dans la recherche? Quelles modifications et quelles stratégies pourraient être adoptées pour faciliter la participation de ces personnes?

7. Comme le chercheur partagera-t-il les résultats de la recherche? 


\section{Annexe 3: Ressources connexes ${ }^{4}$}

Directives et protocoles connexes en matière d'éthique

Canadian Council for Refugees. "Code of Ethics for Groups Involved in the Private Sponsorship of Refugees." 2010. http://ccrweb.ca/en/code-ethics-groups-involved-private-sponsorship-refugees.

European Commission Directorate-General for Research and Innovation. "Guidance Note: Research on Refugees, Asylum Seekers \& Migrants." n.d. http://ec.europa. $\mathrm{eu} / \mathrm{research} / \mathrm{participants/data/ref/h2020/other/hi/}$ guide_research-refugees-migrants_en.pdf.

Human Rights Watch. "About Our Research." n.d. https:// www.hrw.org/about-our-research.

International Labour Organization. "Handbook for ActionOriented Research on the Worst Forms of Child Labour Including Trafficking in Children." 2000. http://www.ilo .org/asia/whatwedo/publications/wCMs_160996/lang-en/ index.htm.

- - - Hard to See, Harder to Count: Survey Guidelines to Estimate Forced Labour of Adults and Children. Geneva: ILO, 2011. http://un-act.org/wp-content/uploads/2015/o6/ Harder-to-See-Harder-to-Count.pdf.

Joint IDP Profiling Service, Danish Refugee Council. "Overview of a Profiling Exercise: 'A Step-by-Step."' n.d. https:// drc.dk/media/1214516/step-by-step-idp-profiling.pdf.

Metropolis British Colombia. "MBC Charter of CommunityUniversity Research Principles." n.d. http://mbc.metropolis.net/assets/uploads/files/MBC_Community_Charter. pdf.

PICUM. "Some Guidelines for Assisting Undocumented Migrants" 2112. http://picum.org/picum.org/uploads/file_ /ethicalguidelinesinenglish.pdf.

unicef. Guidelines on the Protection of Child Victims of Trafficking. New York: UNICEF Technical Notes, 2006. http://www.unicef.org/ceecis/o610-Unicef_Victims_ Guidelines_en.pdf.

World Health Organization. WHO Ethical and Safety Recommendations for Researching, Documenting and Monitoring Sexual Violence in Emergencies. Geneva: wHо, 2007. http://www.who.int/gender/documents/oms_ Ethics\&Safety1oAug07.pdf.

\section{Littérature savante}

Bakewell, Oliver. "Editorial Introduction. Researching Refugees: Lessons from the Past, Current Challenges and Future Directions." Refugee Survey Quarterly 26, no. 3 (2007): 6-14.

Bertrand, Didier. "The Autobiographical Method of Investigating the Psychosocial Wellness of Refugees." In Psychosocial Wellness of Refugees: Issues in Qualitative and
Quantitative Research, ed. F.L. Ahearn, 88-104. New York: Berghahn Books, 2000.

Bloch, Alice. "Carrying Out a Survey of Refugees: Some Methodological Considerations and Guidelines." Journal of Refugee Studies 12, no. 4 (1999): 367-83.

Block, Karen, Elisha Riggs, and Nick Haslam, eds. Values and Vulnerabilities: The Ethics of Research with Refugees and Asylum Seekers. Toowong, Australia: Australian Academic, 2013.

Block, Karen, Deborah Warr, Lisa Gibbs, and Elisha Riggs. "Addressing Ethical and Methodological Challenges in Research with Refugee-Background Young People: Reflections from the Field." Journal of Refugee Studies 26, no. 1 (2013): 69-87.

Bouquet, Brigitte, and Marcel Jaeger. "Tensions entre mise en oeuvre des politiques migratoires et questions éthiquesdu travail social." Hommes et migrations 129 (2011): 10-21.

Clark-Kazak, Christina. "Power and Politics in Migration Narrative Methodology: Research with Young Congolese Migrants in Uganda." Migration Letters 6, no. 2 (2009): $175-82$.

de Haene, Lucia, Hans Grietens, and Karine Verschueren. "Holding Harm: Narrative Methods in Mental Health Research on Refugee Trauma." Qualitative Health Research 20, no. 12 (2010): 1664-76.

Doná, Giorgia. "The Microphysics of Participation in Refugee Research." Journal of Refugee Studies 20, no. 2 (2007): 210-29.

Dyregrov, Kari, Atle Dyregrov, and Magne Raundalen. "Refugee Families: Experience of Research Participation." Journal of Traumatic Stress 13, no. 3 (2000): 413-26.

Ellis, B. Heidi, Maryam Kia-Keating, Siraad Aden Yusuf, Alisa Lincoln, and Abdirahman Nur. "Ethical Research in Refugee Communities and the Use of Community Participatory Methods." Transcultural Psychiatry 44, no. 3 (2007): 459-81.

Hugman, Richard, Linda Bartolomei, and Eileen Pittaway. "Human Agency and the Meaning of Informed Consent: Reflections on Research with Refugees." Journal of Refugee Studies 24, no. 4 (2011): 655-71.

Hugman, Richard, Eileen Pittaway, and Linda Bartolomei. "When 'Do No Harm' Is Not Enough: The Ethics of Research with Refugees and Other Vulnerable Groups." British Journal of Social Work 41, no. 7 (2011): 1271-87.

Jacobsen, Karen, and Loren Landau. "The Dual Imperative in Refugee Research: Some Methodological and Ethical Considerations in Social Science Research on Forced Migration." Disasters 27, no. 3 (2003): 185-206.

- - Researching Refugees: Some Methodological and Ethical Considerations in Social Science and Forced Migration. Geneva: UNHCR, 2003. 
Knight, Lesley-Anne. "Humanitarian Crises and Old Age: Guidelines for Best Practice." Age and Ageing 29, no. 4 (2004): 293-5.

Lammers, Ellen. "Researching Refugees: Preoccupations with Power and Questions of Giving." Refugee Survey Quarterly 26, no. 3 (2007): 72-81.

Leaning, Jennifer. "Ethics of Research in Refugee Populations." Lancet 357, no. 9266 (2001): 1432-3.

Lott, Jason P. "Module Three: Vulnerable/Special Participant Populations." Developing World Bioethics 5, no. 1 (2005): 30-54.

Macchiavello, M. Urban Forced Migrants in Kampala: Methodologies and Ethical and Psychological Issues. Johannesburg, Forced Migration Studies Programme, University of the Witwatersrand, 2003.

Mackenzie, Catriona, Christopher McDowell, and Eileen Pittaway. "Beyond 'Do No Harm': The Challenge of Constructing Ethical Relationships in Refugee Research." Journal of Refugee Studies 20, no. 2 (2007): 299-319.

Mulumba, Deborah. "The Challenges of Conducting Research among Rural-Based Refugees in Uganda." Refugee Survey Quarterly 26, no. 3 (2007): 61-71.

Penz, G. Peter. Ethics and Social Science in the Analysis of Development-Induced Displacement: Issues of Theory and Methodology. Toronto: Centre for Refugee Studies, 2002.

Perry, Kristen H. "Ethics, Vulnerability, and Speakers of Other Languages: How University IRBS (Do Not) Speak to Research Involving Refugee Participants." Qualitative Inquiry 17, no. 10 (2011): 899-912.

Polzer Ngwato, Tara. "Collecting Data on Migrants through Service Provider Ngos: Towards Data Use and Advocacy." Journal of Refugee Studies 26, no. 1 (2013): 144-54.

Refugee Studies Centre, Queen Elizabeth House, University of Oxford. "Ethical Guidelines for Good Research Practice." Refugee Survey Quarterly 26, no. 3 (2007): 162-72. (Adapted from Association of Social Anthropologists, "Ethical Guidelines for Good Research Practice," http:// www.theasa.org/ethics/guidelines.shtml.)

Rodgers, Graeme. 'Hanging Out' with Forced Migrants: Methodological and Ethical Challenges." Forced Migration Review 21 (2005): 48-9.

Rousseau, Cecile, and Laurence Kirmayer. "From Complicity to Advocacy: The Necessity of Refugee Research." American Journal of Bioethics 10, no. 2 (2010): 65-7.

Samaddar, Ranabir. "Power, Fear, Ethics." Refugee Watch 14 (2001): 12-20.

Schweitzer, Robert, and Zachary Steel. Researching Refugees: Methodological and Ethical Considerations in Doing Cross-cultural Research: Ethical and Methodological Perspectives, ed. P. Liamputtong, 87-102. Springer, 2008.
Smith, Valerie J. "Ethical and Effective Ethnographic Research Methods: A Case Study with Afghan Refugees in California." Journal of Empirical Research on Human Research Ethics 4, no. 3 (2009): 59-72.

Spoljar-Vrzina, S.M., S. Martic-Biocina, and M.K. Gilliland. "The Anthropological Approach to the Study of the Uprooted in Croatia: Some Guidelines for Future Projects." Collegium Antropologicum 20, no. 2 (1996): 293-9.

Temple, Bogusia, and Rhetta Moran, eds. Doing Research with Refugees: Issues and Guidelines. Bristol: Policy, 2011.

Thomas, Samantha, and Sarah Byford. "Research with Unaccompanied Children Seeking Asylum." British Medical Journal 327, no. 7428 (2003): 1400.

Tomkinson, Sule. "Doing Fieldwork on State Organizations in Democratic Settings: Ethical Issues of Research in Refugee Decision Making." Forum Qualitative Sozialforschung / Forum: Qualitative Social Research 16, no. 1 (2015).

Yu, Elena S.H., and William T. Liu. "Methodological Problems and Policy Implications in Vietnamese Refugees Research." International Migration Review 20, no. 2 (1986): 483-501.

Zimmerman, Cathy. wHO Ethical and Safety Recommendation for Interviewing Trafficked Women. London: Health Policy Unit, London School of Hygiene \& Tropical Medicine Daphne Programme of the European Commission World Health Organization, 2003.

Zion, Deborah, Linda Briskman, and Bebe Loff. "Returning to History: The Ethics of Researching Asylum Seeker Health in Australia." American Journal of Bioethics 10, no. 2 (2010): 48-56.

\section{Notes}

1 Préparé par Christina Clark-Kazak et adopté par les comités exécutifs du Conseil canadien pour les réfugiés (CCR), de l'Association canadienne détudes sur les réfugiés et la migration forcée (ACERMF) et du Centre pour les études sur les réfugiés de l'Université York (CRS). Les individus suivants étaient sur le comité du projet et ont contribué des idées qui se retrouvent dans ce document : Tanya Aberman, Idil Atak, Michael Casasola, Sherman Chan, Alison Collins-Mrakas, Janet Dench, John Dubé, Sue Grafe, Celia Haig-Brown, Jennifer Hyndman and Michaela Hynie. Nous sommes reconnaissants des contributions des participants de l'atelier à la Consultation d'automne $2016 \mathrm{du}$ CCR, à laquelle Johanna Reynolds et John Carlaw ont également facilité des groupes. Ce document s'inspire également du Code d'éthique sur le parrainage adopté par le CCR. Le financement du projet fut fourni par le Conseil de recherches en sciences sociales; Immigration, réfugiés et citoyenneté Canada; et Refuge : Revue canadienne sur les réfugiés. Nous sommes reconnaissants des contributions en 
nature du CCR, de l'ACERMF, du CRS et du MOSAIC. La traduction vers le français du texte original en anglais fut faite par Marie-Noelle Ecobichon.

2 Dans ce document, le masculin (singulier ou pluriel) est utilisé comme représentant de tous les genres, dans le seul but d'alléger le texte.

3 Les lois antiterroristes ont une vaste portée et peuvent changer. Il est donc important que les personnes qui travaillent dans des contextes de migration forcée tiennent compte de ces lois et de leurs répercussions sur leur travail. Ce document ne valide en aucune façon les lois en vigueur. 4 Tanya Aberman organisa cette section en utilisant la recherche ultérieure de Chizuru Nobe Ghelani.

Christina Clark-Kazak est professeure agrégée à l'École d’affaires publiques et internationales, l'Université d'Ottawa. Son courrielest: cclarkka@uottawa.ca. 